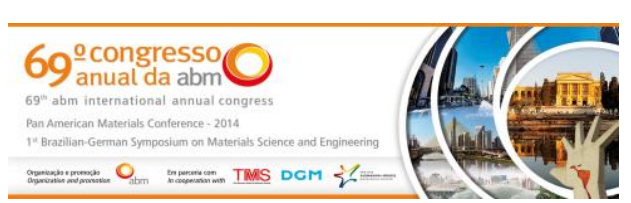

Tema: Engenharia de superfície

\title{
ABLAÇÃO COM LASER DE PULSOS ULTRACURTOS: AVALIAÇÃO DOS EFEITOS NO AÇO AISI 1045*
}

\author{
Denilson de Camargo Mirim ${ }^{1}$ \\ Wagner de Rossi2 \\ Ricardo ElgulSamad2 \\ Nilson Dias Vieira Júnior ${ }^{2}$ \\ Maurício David Martins das Neves ${ }^{2}$
}

\section{Resumo}

Corte, furação e texturização são alguns dos processos que utilizam laser na produção industrial comercial. São processos bem estabelecidos, que contam com equipamentos dedicados e metodologias bem estabelecidas. Apesar de eficientes e de certa precisão, são processos térmicos com inconvenientes intrínsecos como rebarbas, material ressolidificado, zona afetada pelo calor e certa imprecisão dimensional. A utilização de pulsos laser ultracurtos, de largura temporal da ordem de algumas dezenas de femtossegundos torna possível a realização destes processos sem a produção de calor, como no caso de dielétricos, ou com geração desprezível de calor para o caso de metais. Neste trabalho, utilizou-se o aço AISI 1045 para o estudo da interação com pulsos laser de femtosegundos, com o intuito de obter as condições de processo em que a produção de calor seja minimizada ou até mesmo eliminada. Os parâmetros de processo variados foram a largura temporal dos pulsos laser, 25, 87 e 124 fs, a densidade de potência e o número de pulsos sobrepostos. A técnica D-Scan foi utilizada para a determinação dos limiares de ablação em função deste número de pulsos sobrepostos. Foram analisadas a microestrutura das regiões vizinhas à região ablacionada, com o intuito de identificar as possíveis transformações de fase que poderiam estar correlacionadas à produção de calor pelo processo. Condições de mínima produção de calor foram obtidas com minimização de produção de rebarbas e de material ressolidificado.

Palavras-chave: Laser de femtossegundos; Microusinagem; Processos não térmicos; AISI 1045.

\section{LASER ABLATION ULTRASHORT PULSE: EVALUATION OF EFFECTS ON STEEL AISI} 1045

\begin{abstract}
Cutting, drilling and texturing are some of the processes that use laser industrial commercial production. These processes are well established and have dedicated equipment and methodologies well established. Although efficient and with certain precision, are thermal processes with inherent disadvantages such as burrs, material solidified, heat affected zone and some dimensional inaccuracy. The use of ultrashort laser pulses with a temporal width in the order of few tens of femtoseconds makes possible the achievement of these processes without the heat generation, as in the case of dielectrics or with minimal generation of heat for the case of metals.In this work, we used the steel AISI 1045 to study its interaction with femtosecond laser pulses, in order to obtain process conditions where heat generation is minimized or even eliminated.The process parameters varied were the temporal width of the laser pulses, 25, 87 and $124 \mathrm{fs}$, the power density and the number of overlapped pulses. The microstructure of the regions surrounding the ablated region was analyzed to identify possible phase transformations that could be correlated to production of heat by the process. Conditions of minimal heat production were obtained with minimization of production of burrs and resolidified material.

Keywords: Femtosecond laser; Micromachining; Non-thermal processes; AISI 1045.

1 Aluno de Doutorado, Instituto de Pesquisas Energéticas e Nucleares (IPEN), São Paulo, SP, Brasil.

2 Doutor, Instituto de Pesquisas Energéticas e Nucleares (IPEN), São Paulo, SP, Brasil.
\end{abstract}

\footnotetext{
* Contribuição técnica ao 69 Congresso Anual da ABM - Internacional e ao 14ํㅡㄹ ENEMET - Encontro Nacional de Estudantes de Engenharia Metalúrgica, de Materiais e de Minas,21 a 25 de julho de 2014, São Paulo, SP, Brasil.
} 


\section{INTRODUÇÃO}

Ao longo dos últimos anos, a necessidade de tecnologias de micro-usinagem impulsionou o crescimento da pesquisa científica para a indústria. Segmentos de aparelhos médicos, microeletrônica e a demanda do mundo automotivo proporcionaram uma grande variedade de aplicações, tais como micromotores, circuitos microfluídicos, MEMS (Micro Sistema Elétrico Mecânico), dispositivos médicos, ferramentas eletrônicas, filtros de partículas, micromoldes e microválvulas, entre outros [1]. Este enorme crescimento nesse segmento exige uma variedade de novos métodos de microusinagem.

Técnicas para usinar a superfície de materiais estão sendo continuamente melhoradas. Recentemente, microusinagens mecânicas com sistemas de posicionamento precisos e brocas muito pequenas foram usadas para cortar materiais, que abrange o intervalo de alguns milímetros até algumas dezenas de nanômetros [2]. Uma técnica semelhante, que também se aplica à microusinagem direta e requer um sistema de posicionamento de alta precisão, mas sem contato, é a microusinagem por desacarga de elétrons (EDM), em que o material é removido por um eletrodo guiado ao longo do percurso desejado, muito perto da superfície de um material condutor imerso em um fluido dielétrico. Neste processo, uma faísca é estabelecida entre o eletrodo e o material a ser processado, a remoção da matéria se dá por fusão e evaporação [3,4], oferecendo uma resolução micrométrica.

A Fotolitografia, é outra técnica de microusinagem que está disponível para a fabricação de circuitos integrados desde 1970, foi melhorada, e hoje em dia é usada para criar estruturas que variam de micrometros, como no caso dos dispositivos MEMS, até dezenas de nanômetros para circuitos integrados [5]. Esta técnica remove seletivamente o material a partir de películas finas (de nitreto / óxido dePolissilício) depositados sobre substratos de silício, e também a partir da massa do substrato [6]. A fotolitografia permite a produção de lotes, mas é considerado um método indireto, uma vez que o material não é removido por uma ferramenta.

Embora amplamente utilizada, as técnicas descritas acima têm algumas desvantagens, tais como o desgaste da ferramenta (micro-usinagem mecânica e EDM) e alta complexidade (fotolitografia). Além disso, na usinagem de materiais diferentes, as taxas de erosão podem depender muito do material e da ferramenta, dos parâmetros de processo, ou ainda necessitam de troca da ferramenta. Além disso, a técnica pode ser restrita a uma classe de materiais específicos, tais como os metais, no caso EDM. Outras limitações incluem a pequena velocidade de ataque, nas técnicas que exigem o posicionamento preciso (Usinagem mecânica, EDM), impedindo o processamento em lote, e da alta complexidade da fotolitografia, o que não permite prototipagem rápida.

Em vista das limitações citadas acima, o laser de pulsos ultracurtos surge como uma ferramenta valiosa para microusinagem. Apenas alguns anos depois da invenção do laser, ele já estava sendo usado como uma ferramenta para ablacionar [7] e usinar [8], uma ampla variedade de materiais, incluindo metais [9], dielétricos [10], semicondutores [11], compostos [12], e tecidos biológicos [13].Nestes processos, a usinagem é baseada em processos térmicos resultantes do aquecimento de material pelo laser [14], e a ablação ocorre em consequência da fusão e vaporização resultantes das transições de fase devido ao calor depositado no material processado $[15,16]$.

$\mathrm{Na}$ usinagem com pulsos laser, o feixe é focado na superfície do material a ser processado, a luz é absorvida e o material sofre uma mudança física em torno da

\footnotetext{
* Contribuição técnica ao $69^{\circ}$ Congresso Anual da ABM - Internacional e ao 14ํㅡㄹ ENEMET - Encontro Nacional de Estudantes de Engenharia Metalúrgica, de Materiais e de Minas,21 a 25 de julho de 2014, São Paulo, SP, Brasil.
} 
região de absorção. As dimensões das estruturas que podem ser usinadas dependem, em uma primeira aproximação, do diâmetro do feixe focado. Para um feixe Gaussiano TEMoo limitado por difração, [17] este diâmetro pode ser da ordem do comprimento de onda do laser, variando desde algumas centenas de nanômetros (UV lasers Excimer até algumas dezenas de micrometros (laser $\mathrm{CO}_{2}$ ). No entanto, mesmo que o ponto de laser seja o menor possível sobre a superfície do material, a região de interação resulta em um volume muito maior devido à difusão de calor gerado no processo. Para pulsos longos, que duram mais do que o período de transferência de calor dos elétrons para a rede cristalina do material (cerca de dezenas de picosegundos), a dinâmica da interação laser-matéria depende dos parâmetros do laser (comprimento de onda, energia do pulso, taxa de repetição) e das características físicas (absorção, capacidade térmica, condutividade térmica) do material a ser usinado. Neste regime de pulsos longos, o processo de usinagem é uma consequência do aquecimento (que resulta na fusão, evaporação, sublimação), dependendo da energia absorvida por unidade de tempo e de volume e como ele flui dentro do material. Quando a radiação laser é absorvida, o material é aquecido e processos termodinâmicos são responsáveis pela difusão de calor e transformações de fases. À medida que a duração do pulso é diminuída, a propagação espacial de calor é diminuída e transformações de fase que levam à remoção de material ocorrem de forma mais eficiente. Neste regime, a extensão da propagação do calor (do seu efeito) diminui com a raiz quadrada da duração do pulso, demonstrando que a ablação é um processo térmico [18]. O calor em excesso que não é usado para a ablação do material flui para o ambiente, para aumentar a temperatura, e para o interior do material, criando uma zona afetada pelo calor (ZAC), no qual as transições de fase modificam as propriedades do material, geralmente, de uma forma prejudicial. Como consequência, as estruturas menores que requerem pulsos curtos para serem gravados, produzem também uma reduzida ZAC. No entanto, embora pulsos de microssegundo e pulsos de sub-nanosegundos laser gerem intensidades altas o suficiente para usinar metais, semicondutores e mesmo alguns dielétricos transparentes, a condução de calor expande a região afetada para além do diâmetro do feixe focado, cria uma ZAC grande, e pode também gerar estruturas várias vezes maiores do que o comprimento de onda utilizado. Efeitos colaterais de calor, como de rebarbas, detritos, e material fundido, tendem a aumentar a área de interação e limitar a precisão de usinagem. Além disso, embora as intensidades elevadas atingíveis neste regime permitam a usinagem de materiais transparentes tais como sílica e safira, grandes quantidades de energia são necessários para atingir o limiar de ablação, resultando em tensões térmicas e danos, tais como lascas e microrachaduras [19,20]. Para lidar com esses problemas, diferentes comprimentos de onda são necessários para a absorção eficiente de materiais diferentes. Para dielétricos, lasers com comprimentos de onda na região do UV são utilizados para melhorar a absorção; alguns vidros, contudo, têm maior absorção no infravermelho distante [21] e, portanto, lasers de $\mathrm{CO}_{2}$ são frequentemente utilizados nestes casos.

Assim, pulsos de femtosegundos $\left(10^{-15} \mathrm{~s}\right)$ surgem como uma nova possibilidade para microusinagem com algumas vantagens sobre os pulsos mais longos. A característica morfológica de uma superfície usinada por estes pulsos ultracurtos decorre diretamente da duração muito curta, que é menor do que o período de vibração térmica da rede cristalina. Isto leva a uma transferência de energia para o material extremamente pequena, que por sua vez resulta em efeitos térmicos minimizados, diminuindo ou eliminando a ZAC e conservando as propriedades

\footnotetext{
* Contribuição técnica ao $69^{\circ}$ Congresso Anual da ABM - Internacional e ao 14ํㅡㄹ ENEMET - Encontro Nacional de Estudantes de Engenharia Metalúrgica, de Materiais e de Minas,21 a 25 de julho de 2014, São Paulo, SP, Brasil.
} 


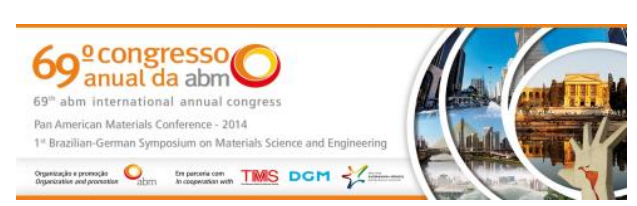

físicas e metalúrgicas do material circundante. Além disso, a duração ultracurta dos pulsos cria intensidades muito elevadas que promovem uma interação altamente não-linear com a matéria, resultando em ablações com precisão nanométrica, e permitindo a microusinagem precisa de estruturas diminutas. Além disso, os pulsos ultracurtos promovem uma ablaçãonão-seletiva em relação à classe do material (dielétrico, metálico, etc), permitindo a usinagem de todos os tipos de materiais, com o mesmo laser. A capacidade de pulsos ultracurtos para usinar qualquer material, mesmo os mais transparentes, sem alterar o comprimento de onda do laser e a recente disponibilidade de sistemas relativamente baratos e estáveis e de alta potência média, que aumentam a velocidade de processamento, está fazendo desses pulsos ferramentas muito versáteis para processar materiais para muitas aplicações de alta tecnologia e dispositivos práticos. As desvantagens para a microusinagem com pulsos de femtosegundos vêm do alto grau de precisão exigido; em sistemas de posicionamento com 3 eixos. Além disso, as baixas potências médias dificultam a produção de lotes, que, contudo vem sendo superado por sistemas modernos, com dezenas de watts, permitindo uma melhoria de rendimento aumentado. A invenção, na segunda metade da década de 1980, de amplificadores de pulso [22,23], o método de Kerr Lens Modelocking (KLM) do laser de Ti: Safira [24], e o bombeamento de lasers de estado sólido por lasers de diodo [25] disseminou o uso de pulsos ultracurtos com energia da ordem de microjoule-milijoule $(\mu \mathrm{J}-\mathrm{mJ})$. A ampla disponibilidade destes sistemas nas décadas de 1990-2000 resultou no surgimento de diversas novas aplicações, incluindo a usinagem por pulsos ultracurtos. Os lasers mais utilizados hoje em dia de pulsos ultracurtos para usinagem são Ti: Safira e Yb: fibra. Os sistemas de Ti: Safira podem entregar pulsos tão curtos quanto algumas dezenas de femtosegundos, centrados em $800 \mathrm{~nm}$, com energias da ordem de $\mathrm{mJ}$, e as taxas de repetição de alguns $\mathrm{kHz}$. Os lasers de itérbio geram pulsos centrados em torno de $1030 \mathrm{~nm}$ com centenas de femtosegundos, e, embora limitado em energia para a faixa de $\mu \mathrm{J}$, operam em dezenas de $\mathrm{MHz}$ de taxas de repetição, aumentando sua velocidade de usinagem. Desenvolvimentos recentes na tecnologia laser oferecem projetos de sistemas de alta potência média, que em conjunto com novos dispositivos [26] e com sistemas de digitalização de alta velocidade permitem a utilização de pulsos ultracurtos para usinagem na produção de lotes, sem limitar a sua capacidade de prototipagem rápida.

\section{MATERIAIS E MÉTODOS}

Este trabalho utilizou a técnica de varredura na diagonal (D-Scan) [27,28], em vez do tradicional método do "dano zero" [29], para medir o limiar de ablação como função dos pulsos sobrepostos N. Nesta técnica, a amostra é colocada com a sua superfície ortogonal à direção de propagação de um feixe Gaussiano TEMoo focalizado, e é deslocada em duas direções ao mesmo tempo, paralelae perpendicular ao eixo do feixe. Numa posição próxima à cintura do feixe, um perfil é gravado como mostrado na Figura 1. A região de ablação apresenta uma largura mínima na posição de focagem e dois lobos máximos com largura $2 \rho_{\max }$, simetricamente localizadas antes e depois da cintura do feixe.

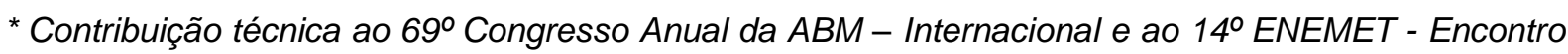
Nacional de Estudantes de Engenharia Metalúrgica, de Materiais e de Minas,21 a 25 de julho de 2014, São Paulo, SP, Brasil.
} 

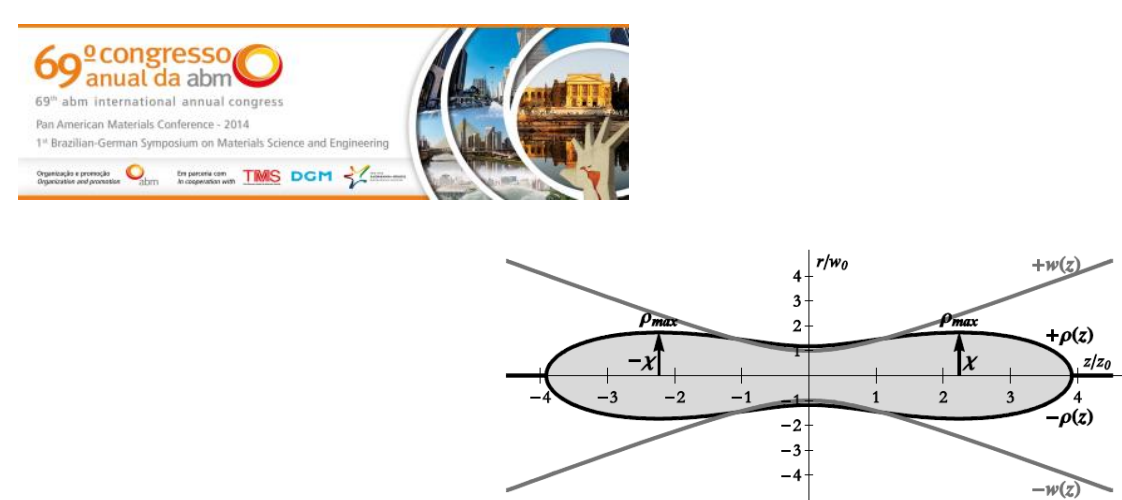

Figura 1. Perfil gravado na superfície da amostra que tem dois máximos de lobos transversais na posição $X$ com o máximo de dimensão $2 \rho_{\max }$. As linhas $+W(z)$ e $W$ - $(z)$ representamo perfil do feixe laser onde a intensidade é de $1 / \mathrm{e}^{2}$ do seu valor de pico.

Se não há nenhum efeito de calor importante, e a experiência é realizada acima de certa intensidade, pode ser mostrado [27] que, para um feixe Gaussiano TEMoo, o limiar de ablação está diretamente relacionado com a dimensão máximapmax e os pulsos laser de energia $\mathrm{E}_{0}$, através da própria expressão:

$$
F_{t h}=\frac{E_{0}}{e \pi \rho_{\max }^{2}} \cong 0.117 \frac{E_{0}}{\rho_{\text {max }}^{2}},(1)
$$

Para levar em conta os efeitos de incubação, a sobreposição de $\mathrm{N}$ pulsos diferentes é considerada como a razão entre a soma das intensidades produzidas em $\left(X, \rho_{\max }\right)$ por cada pulso que atinge a amostra, durante o seu movimento, e a intensidade do pulso centrado gerado em $(X, 0)$. Partindo deste pressuposto, pode ser mostrado [30] que:

$$
N=\vartheta_{3}\left(0, e^{-\left(\frac{v_{y}}{f \rho_{\max }}\right)^{2}}\right)
$$

Onde $\vartheta_{3}$ é a função elíptica do terceiro tipo, teta de Jacobi, f é a frequência de repetição do laser e $v_{y}$ é a velocidade de translação transversal da amostra. Para as taxas de repetição transversais e velocidades baixas, a eq. 2 pode ser aproximada para:

$$
\mathrm{N} \sim 1.8 \rho_{\max } / v_{\mathrm{y}},(3)
$$

O material utilizado para a investigação da interação com pulsos ultracurtos foi o aço carbono AISI 1045, por apresentar pontos bem definidos de fusão, $1410^{\circ} \mathrm{C}$ e de transição de fase Ferrita-Austenita na temperatura de $704^{\circ} \mathrm{C}$, o que permite uma monitoração indireta das temperaturas por eventuais mudanças microestruturais. Este material tem a composição química apresentada na tabela 1. A amostras utilizadas tinham espessuras de aproximadamente $1 \mathrm{~mm}$ e a área irradiada era de geometria retangular de $15 \times 20 \mathrm{~mm}$, que em sua preparação foram embutidas em baquelite, lixadas e posteriormente polidas com pasta de diamante de granulação de $1 \mu \mathrm{m}$.

Tabela 1. Composição química do AISI 1045

\begin{tabular}{l|l|l|l|l|l}
\hline$\%$ Peso & C & Mn & Fe & S & P \\
\hline AISI 1045 & $0,43-0,5$ & $0,6-0,90$ & bal. & 0,05 & $0,045(\max )$ \\
\hline
\end{tabular}

O experimento foi conduzido com um sistema laser de Ti: Safira (FemtopowerCompact Pro CE-Phase HP / HR de Femtolasers), com emissão centrada em $785 \mathrm{~nm}$, com $37 \mathrm{~nm}$ de largura de banda, e taxa de repetição máxima de $4 \mathrm{kHz}$ e mínima de $100 \mathrm{~Hz}$, controlada por uma célula de Pockels, e $800 \mu \mathrm{J}$ de energia máxima por pulso,e emissão polarizada linearmente na direção horizontal.

\footnotetext{
* Contribuição técnica ao 69ำ Congresso Anual da ABM - Internacional e ao 14ํㅡㄹ ENEMET - Encontro Nacional de Estudantes de Engenharia Metalúrgica, de Materiais e de Minas,21 a 25 de julho de 2014, São Paulo, SP, Brasil.
} 


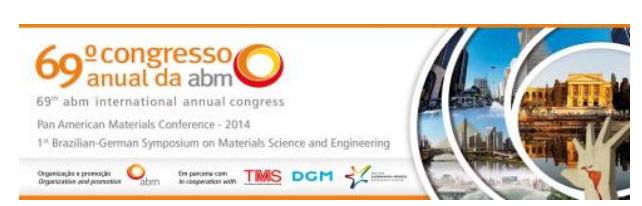

Três durações temporais diferentes de pulsos foram utilizadas: 25 , 87 e 124 fs. Para as irradiações, as energias dos pulsos foram atenuadas para aproximadamente $60 \mu \mathrm{J}$, o feixe foi focado por uma lente de comprimento focal de 75 milímetros (Thorlabs AC254 - 075 - B ), e a amostra foi movida por um sistema de 3 eixos controlado por computador. Durante o experimento, a amostra foi sempre movida de antes para depois da cintura do feixe, isto é, no início o foco se encontrava no interior da amostra, movimentando-se da direita para a esquerda, terminando 0 movimento com o foco acima da sua superfície. Várias combinações de taxas de repetição e velocidades de deslocamento transversal da amostra vy (no plano horizontal) foram utilizadas para cobrir as superposições de um único pulso até mais de $10^{4}$ pulsos. Assim foram utilizadas as taxas de repetição de 100,500 e $4000 \mathrm{~Hz}$ e velocidades de 0,$05 ; 0,1 ; 0,2 ; 0,3 ; 0,4 ; 0,5 ; 1 ; 2 ; 2,5 ; 3 ; 5 ; 10 ; 20$ e $30 \mathrm{~mm} / \mathrm{s}$. A velocidade de deslocamento longitudinal da amostra, vz (no plano horizontal), era sempre igual a vy para gravar perfis alongados [30]. Após a gravação de cada traço D-Scan, a amostra foi deslocada verticalmente (direção x) por 400 micrômetros, esta separação foi usada como uma escalada para medir $2 \rho$ máximo no microscópio óptico (MO). A Figura 2 apresenta traços obtidos no experimento de $25 \mathrm{fs}$.

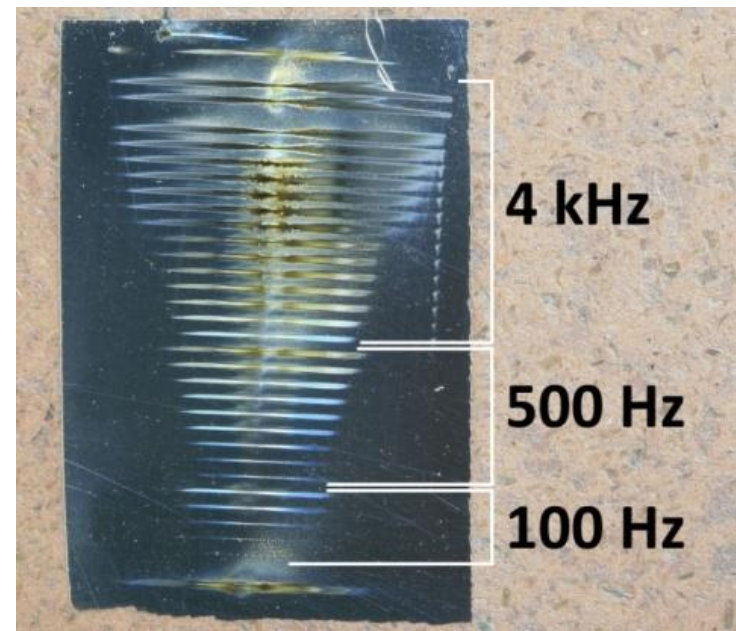

Figura 2. Traços gerados pela técnica D-Scan no experimento com pulsos de $25 \mathrm{fs}$

Esses pulsos foram usados para medir o limiar de ablação ( $F_{\text {th }}$ ) para pulsos sobrepostos e o parâmetro de incubação (k) do aço AISI 1045, assim como para verificação dosseus efeitos na morfologia da superfície em função da variação dos parâmetros do experimento. Todas as irradiações foram realizadas em atmosfera protetora de Argônio (Ar) com fluxo de 10L/min.Posteriormente as amostras foram limpas com álcool isopropílico para remover detritos da ablação depositados.

A imagem obtida no microscópio eletrônico de varredura TM3000, apresentada na Figura 3, mostra os seis primeiros traços gerados pela técnica D-Scan, no experimento onde foi usada a largura temporal do pulso de $25 \mathrm{fs}$, com velocidades de varredura crescentes de cima para baixo (na figura 2). A figura da direita mostra o processo de medição dos traços.

\footnotetext{
* Contribuição técnica ao $69^{\circ}$ Congresso Anual da ABM - Internacional e ao 14ํㅡㄹ ENEMET - Encontro Nacional de Estudantes de Engenharia Metalúrgica, de Materiais e de Minas,21 a 25 de julho de 2014, São Paulo, SP, Brasil.
} 

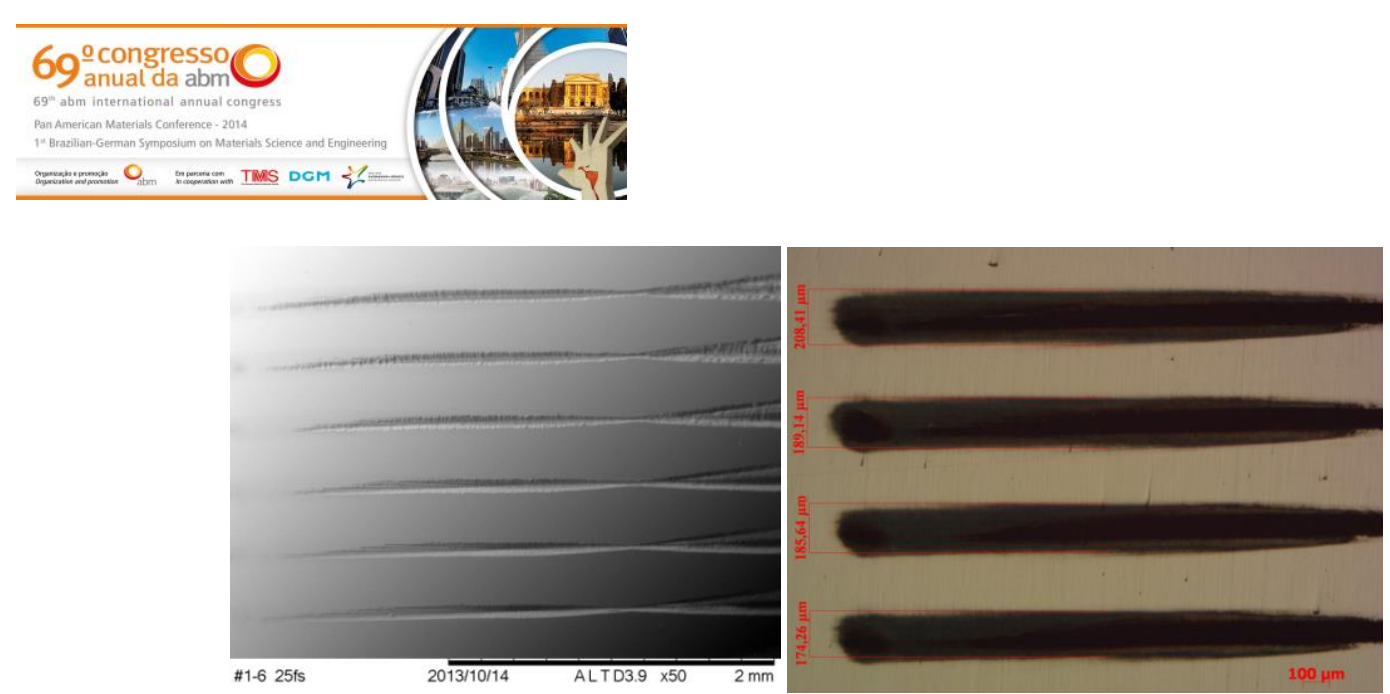

Figura 3. Na imagem da esquerda obtida no MEV, são mostrados os seis primeiros traços gerados pela técnica D-Scan. A imagem da direita obtida no $\mathrm{MO}$, mostra medida $2 \rho_{\max }$ usada no cálculo do limiar de ablação do AISI 1045.

\section{RESULTADOS E DISCUSSÃO}

Nas análises dos traços, foram utilizadas a microscopia óptica (MO) e a microscopia eletrônica de varredura (MEV). As imagens geradas foram utilizadas para se fazer as medidas das larguras dos traços (MO) e a análise das mudanças na morfologia dos traços na superfície do aço (MEV).

No gráfico da Figura 3, observa-se o decréscimo do limiar de ablação $\left(\mathrm{F}_{\text {th }}\right)$ do aço AISI 1045 com o aumento da sobreposição $\mathrm{N}$ de pulsos e um comportamento similar para as três larguras temporais utilizadas.

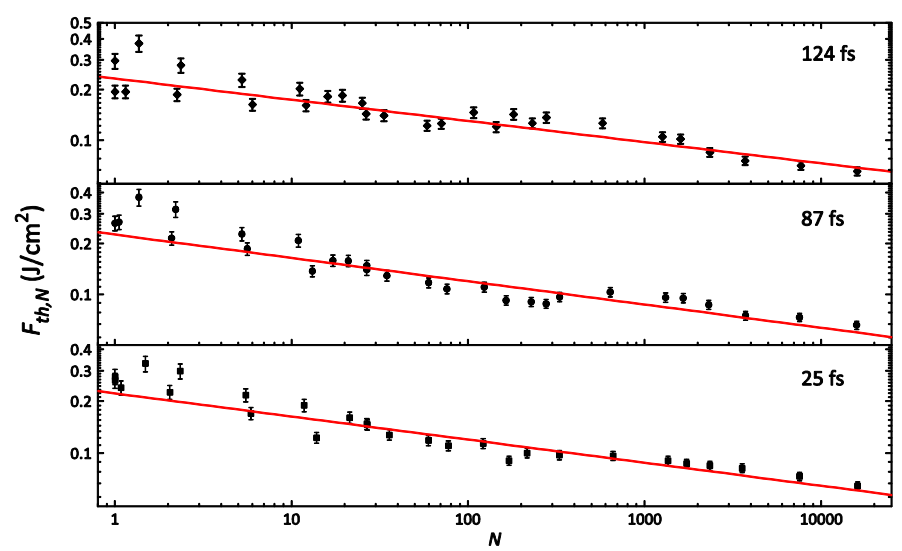

Figura 3. Gráfico do limiar de ablação ( $\left.F_{\text {th }}\right)$ em função do número de pulsos sobrepostos $(N)$ obtido na execução dos traços no aço AISI 1045.

Aproveitando a vantagem da técnica D-Scan, de proporcionar a reprodução rápida de diversas condições com diferentes morfologias de ablação, dividimos 0 experimento em três grupos principais de regimes de ablação, para as três larguras temporais. O primeiro grupo está compreendido entre o primeiro e o sexto traço, na qual a sobreposição está aproximadamente entre 1000 e 15000 pulsos, onde ocorre a ablação mais efetiva (de maior volume) do material. A Figura 4 apresenta o traço realizado na amostra com a maior sobreposição de pulsos. Ao contrário do que se poderia esperar, observa-se que a região onde o foco está na superfície da amostra não corresponde à maior profundidade de ablação. Este fato também se repete para condições de menor sobreposição. Por ser a região onde a intensidade é maior, esperava-se uma maior remoção de material e maior profundidade de usinagem. Um dos fatores que podem ter contribuído para esta diminuição de eficiência na

\footnotetext{
* Contribuição técnica ao 69ำ Congresso Anual da ABM - Internacional e ao 14ํㅡㄹ ENEMET - Encontro Nacional de Estudantes de Engenharia Metalúrgica, de Materiais e de Minas,21 a 25 de julho de 2014, São Paulo, SP, Brasil.
} 
remoção de material é que a alta intensidade produz uma ionização muito grande da atmosfera neste ponto. O plasma formado por esta ionização absorve parte do próprio feixe laser diminuindo a energia que chega até a superfície. Para regiões fora do foco, o diâmetro do feixe é maior,a intensidade é menor e o plasma passa a ser muito pequeno ou inexistente. A eficiência de remoção passa a ser maior quando não há plasma, e com o deslocamento do feixe para posições mais afastadas do foco, a profundidade de ablação passa por um máximo e depois decresce continuamente até não haver mais ablação. O estudo mais detalhado da relação entre intensidade e remoção de material ainda está sendo estudada e deverá resultar em uma otimização de processos com lasers de pulsos ultracurtos.

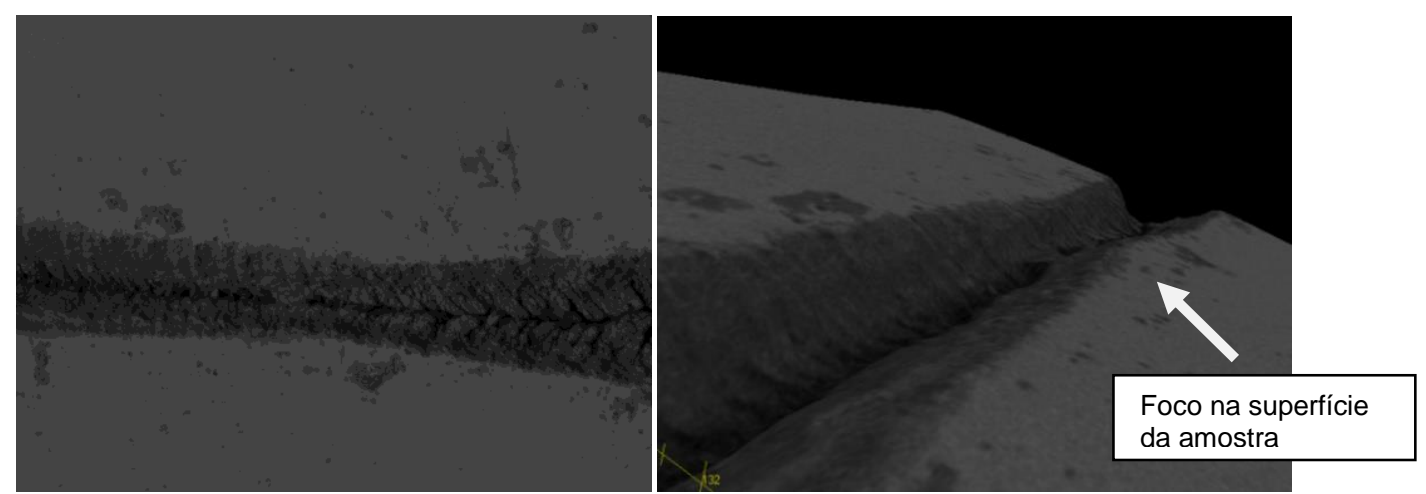

Figura 4. Condição de maior sobreposição de pulsos na execução dos traços no aço AISI 1045.

A partir da observação dos traços realizados nos experimentos, a condição de melhor acabamento, principalmente de borda, foi obtida no traço 6, na largura temporal de $25 \mathrm{fs}$, onde a sobreposição foi de $\mathrm{N} \sim 1280$ pulsos, Figura 5.

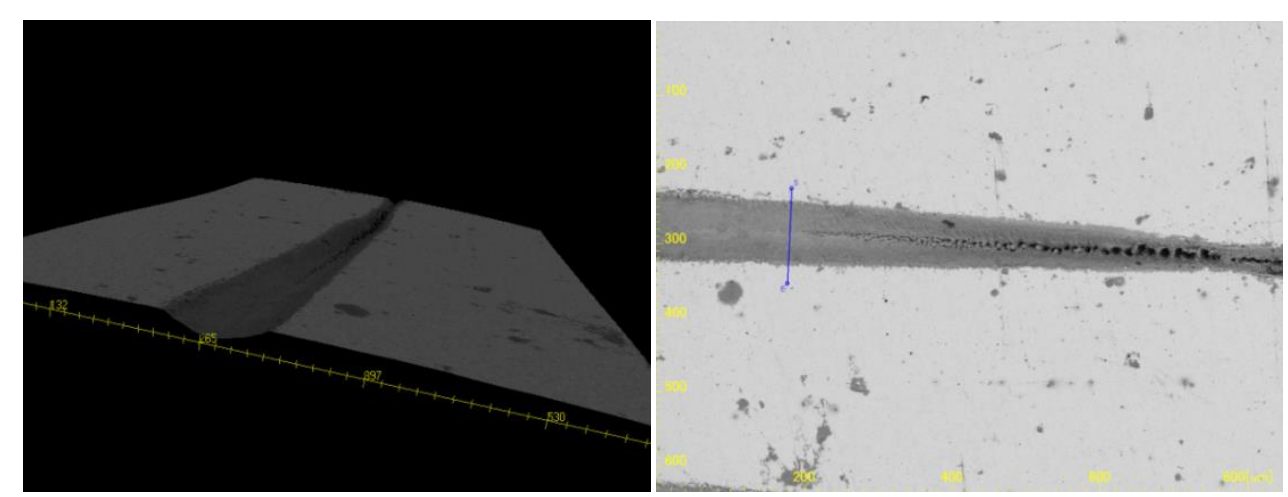

Figura 5. Condição na qual se obteve a borda melhor definida na execução dos traços no aço AISI1045

O segundo grupo está compreendido entre o sétimo e o décimo primeiro traço, em que a sobreposição está aproximadamente entre 50 e 600 pulsos, condição na qual ocorre a remoção de pequenas porções de material que formam uma estrutura granulada, Figura 6. Moradi et al [31], obtiveram resultado similar a essa estrutura no estudo da rugosidade e superhidrofibicidade do aço inoxidável 316L.

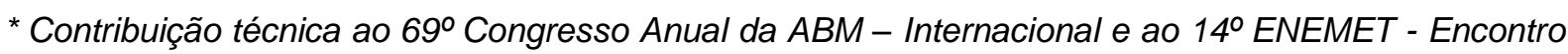
Nacional de Estudantes de Engenharia Metalúrgica, de Materiais e de Minas,21 a 25 de julho de 2014, São Paulo, SP, Brasil.
} 

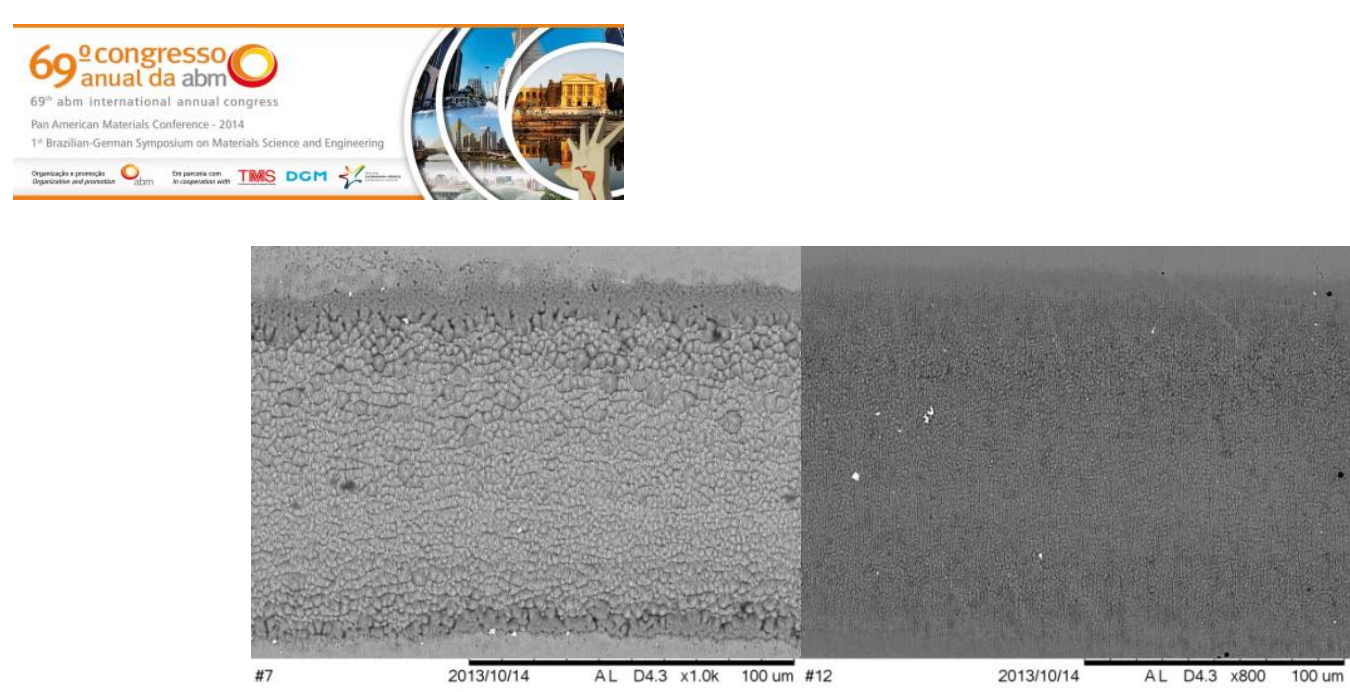

Figura 6. Condições em que se obtém estrutura granulada sem a ablação mais intensa.

O terceiro grupo está compreendido entre um único pulso e a sobreposição de aproximadamente 30 pulsos. Nesta condição ocorre a formação de pequenas ondulações periódicas na superfície do material, com período de aproximadamente $600 \mathrm{~nm}$, e que não variam em função da maior ou menor sobreposição de pulsos. Oberva-se, apenas uma maior profundidade destas estruturas com a maior sobreposição, alterando a coloração do material que se torna mais escurecido, Figura 7. Em estudo sobre a formação de micro/nano estruturas na superfície do silício, Barada K. et al [32], descrevem a formação de estruturas semelhantes às obtidas nesse terceiro padrão obtido no AISI 1045.

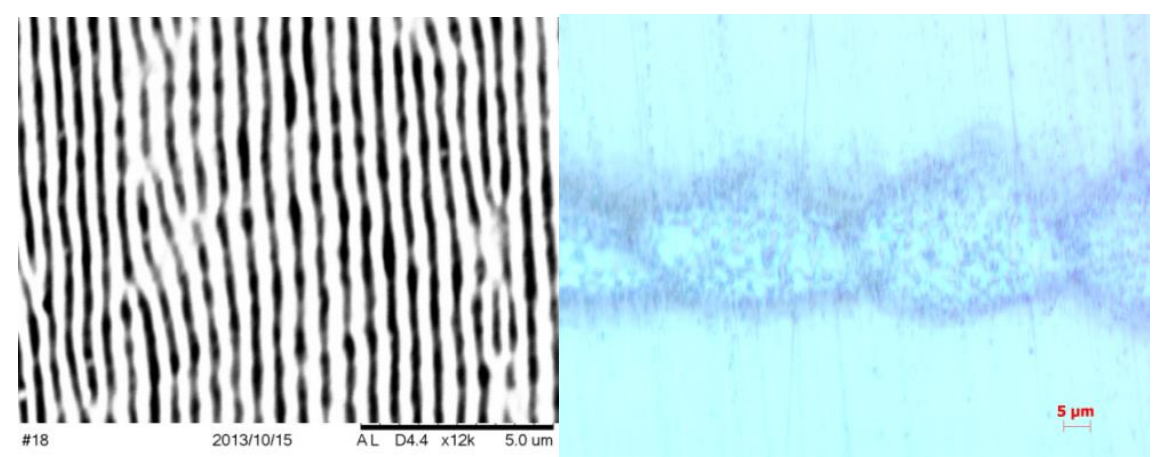

Figura 7. Condições em que se obtém estrutura ondulada e mudança de coloração na superfície irradiada. À esquerda sobreposição de 24 pulsos e à direita pulsos únicos.

Também foram feitas micrografias na superfície das amostras, que após ataque químico com nital a 3\%, não mostraram indícios de propagação de calor fora da região ablacionada, mesmo nas maiores sobreposições de pulsos, conforme mostra Figura 8.

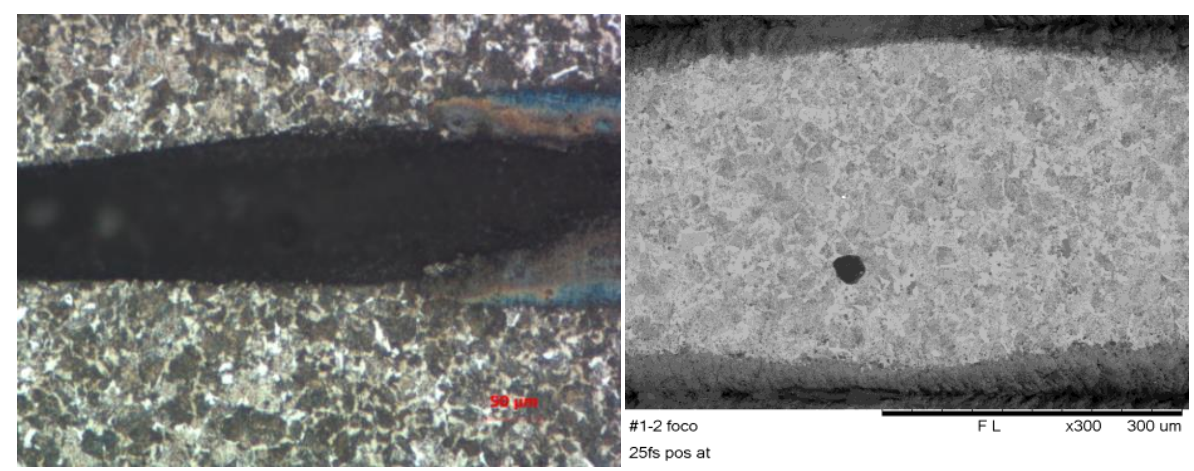

Figura 8. À esquerda traço 6 de melhor acabamento de borda com destaque para grãos que foram cortados pelo laser. À direita, microestrutura inalterada entre os traços de maior sobreposição de pulsosobtida no MEV.

* Contribuição técnica ao $69^{\circ}$ Congresso Anual da ABM - Internacional e ao 14ํㅡㄹ ENEMET - Encontro Nacional de Estudantes de Engenharia Metalúrgica, de Materiais e de Minas,21 a 25 de julho de 2014, São Paulo, SP, Brasil. 


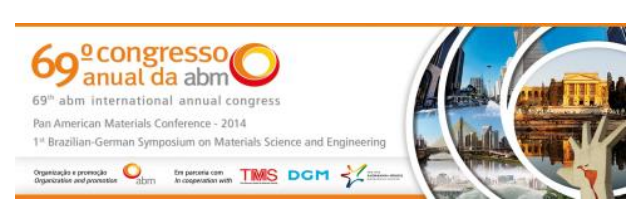

Nas menores sobreposições de pulsos, na condição de formação das ondulações, embora haja a mudança morfologia da superfície, não ocorre retirada de material suficiente para alteração da microestrutura como mostra a Figura 9.

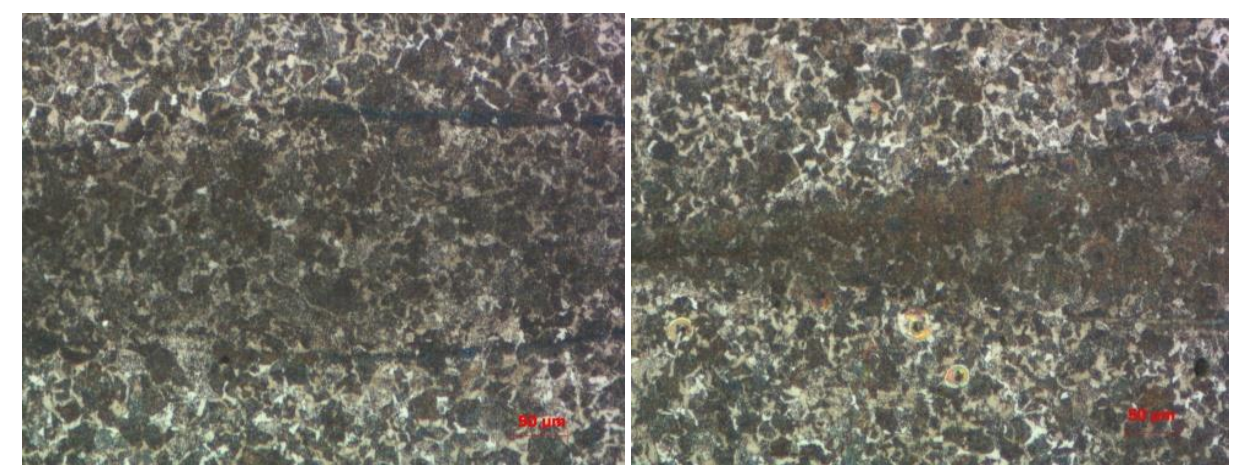

Figura 9. A esquerda sobreposição de 10 pulsos. A direita mesmo traço próximo ao foco.

\section{CONCLUSÃo}

Com o uso da técnica D-Scan foi possível obter uma grande quantidade morfologias na superfície do aço AISI 1045. Os dados referentes às medidas dos traços evidenciaram que o limiar de ablação (Fth) diminui de maneira similar para as três larguras temporais estudadas em função da sobreposição de pulsos. Também foi possível identificar três distintas morfologias de ablação na superfície do aço, com uma ablação mais severa na sobreposição entre 1000 e 15000 pulsos, uma formação granulada entre 50 e 600 pulsos e a formação de ondulações periódicas entre 1 e 30 pulsos. Nessa última condição não ocorre mudança microestrutural no aço, porém ocorre o seu escurecimento, possibilitando o uso desses parâmetros para marcações na superfície deste aço. Verificou-se também que, à medida que se diminui a largura temporal dos pulsos ocorre uma melhoria nas bordas dos traços, sendo a largura temporal de $25 \mathrm{fs}$ a mais adequada para a produção de cortes precisos neste aço.

Após ataque químico, mesmo nas maiores sobreposições, que superaram os 15000 pulsos, não foi identificada uma ZAC, portanto confinando o efeito térmico na região ablacionada.

A ablação com pulsos laser ultracurtos no aço AISI 1045 apresentou, nas diversas morfologias obtidas, promissores resultados, que poderão ser usados na microusinagem de precisão, na gravação e marcação deste material.

\section{Agradecimentos}

Agradeço ao Ipen por disponibilizar toda a infraestrutura necessária para se realizar esse trabalho e ao IFSP-Campus de Itapetininga, pelo uso do seu laboratório de materiais, onde foram preparadas as amostras para esse trabalho.

\section{REFERÊNCIAS}

1 Gad-el-Hak M MEMS : introduction and fundamentals : the MEMS handbook. Boca Raton: CRC Press/Taylor \& Francis. 2006

2 Dornfeld D, Min S, Takeuchi Y. Recent Advances in Mechanical Micromachining.CIRP Ann.-Manuf. Techn. 2006; 55: 745-768.

\footnotetext{
* Contribuição técnica ao $69^{\circ}$ Congresso Anual da ABM - Internacional e ao 14ํㅡㄹ ENEMET - Encontro Nacional de Estudantes de Engenharia Metalúrgica, de Materiais e de Minas,21 a 25 de julho de 2014, São Paulo, SP, Brasil.
} 


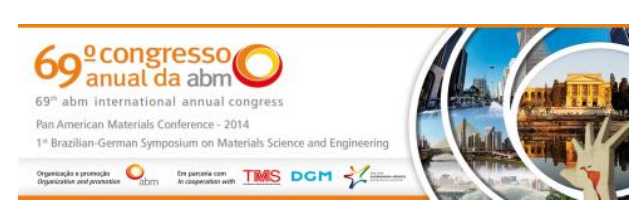

3 Pradhan B, Masanta M, Sarkar B, Bhattacharyya B. Investigation of electrodischarge micro-machining of titanium super alloy. Int. J. Adv. Manuf. Tech. 2009; 41: 1094-1106.

4 Kaminski PC, Capuano MN. Micro hole machining by conventional penetration electrical discharge machine. Int. J. Mach. Tool Manu. 2003; 43: 1143-1149.

5 Chou SY, Krauss PR, Renstrom PJ Imprint lithography with 25-nanometer resolution. Science. 1996; 272: 85-87.

6 Kovacs GTA, Maluf NI, Petersen KE. Bulk micromachining of silicon. Proceedings of the IEEE. 1998; 86: 1536-1551.

7 Honig RE, Woolston JR. Laser-Induced Emission of Electrons, Ions, and Neutral Atoms from Solid Surfaces. Appl. Phys. Lett, 1963; 2: 138-139.

8 Boot HAH, Clunie DM, Thorn RSA Micromachining with a pulsed gas laser. Electron. Lett. 1966; 2: 1.

9 Anisimov SI. Vaporization of Metal Absorbing Laser Radiation. Sov. Phys. JETPUSSR. 1968; 27: 182-183.

10 Kocher E, Tschudi L, Steffen J, Herziger G Dynamics of laser processing in transparent media IEEE J. Quantum Elec. QE. 1972; 8: 120-\&.

11 Bourg H, Frederick RW. Laser Machining of Silicon. J. Electrochem. Soc. 1975; 122: 260-260.

12 Longfell J. High Speed Drilling in Alumina Substrates with a CO2 Laser. AmCeram Soc Bull. 1971; 50: 251-\&.

13 McGuff PE, Deterling RA, Gottlieb LS, Bushnell D, Fahimi HD. Surgical Applications of Laser. Ann. Surg. 1964; 160: 765-\&.

14 Cohen MI, Unger BA, Milkosky JF. Laser Machining of Thin Films and Integrated Circuits. AT\&T Tech. J. 1968; 47: 385-405.

15 Steen WM. Laser material processing. New York: Springer; 2003.

16 Ion JC Laser processing of engineering materials : principles, procedure and industrial application. Oxford: Elsevier/Butterworth-Heinemann; 2005.

17 Kogelnik H, Li T. Laser Beams and Resonators. Appl. Opt. 1966; 5: 1550-1567.

18 Stuart B, Feit M, Rubenchik A, Shore B, Perry M. Laser-Induced Damage in Dielectrics with Nanosecond to Subpicosecond Pulses. Phys. Rev. Lett. 1995; 74: 2248-2251.

19 Lan B, Hong MH, Ye KD, Wang ZB, Chong TC. Laser microfabrication of glasssubstrates by pocket scanning. In: Miyamoto I, Ostendorf A, Sugioka K, Helvajian $\mathrm{H}$, Laser Pulses - Theory, Technology, and Applications editors. Fourth International Symposium on Laser Precision Microfabrication. 2003; 168: 133-136.

20 Ben-Yakar A, Byer RL. Femtosecond laser machining of fluidic microchannels forminiaturized bioanalytical systems. In: Sugioka K, Gower M C, Haglund RF, Pique A,Dubowski JJ, Hoving W, editors. Photon Processing in Microelectronics and Photonics. 2002: 212-217.

21 Lane DW The optical properties and laser irradiation of some common glasses. J. Phys. D Appl. Phys. 1990; $23: 1727$.

22 Strickland D, Mourou G. Compression of amplified chirped optical pulses. Opt. Commun. 1985; 56: 219-221.

23 Diels JC, Rudolph W. Ultrashort laser pulse phenomena : fundamentals, techniques, and applications on a femtosecond time scale. Burlington, MA: Academic Press. 2006.

24 Sibbett W, Lagatsky AA, Brown CTA. The development and application of femtosecond laser systems. Opt. Expr. 2012; 20: 6989-7001.

25 Keller U. Ultrafast solid-state laser oscillators: a success story for the last 20 years with no end in sight. Appl. Phys. B-Las. Opt. 2010; 100: 15-28.

26 Russbueldt P, Mans T, Weitenberg J, Hoffmann HD, Poprawe R. Compact diodepumped 1.1 kW Yb: YAG Innoslab femtosecond amplifier. Opt. Lett., 2010; 35: 41694171.

27 Samad RE and Vieira ND, Geometrical method for determining the surface damage threshold for femtosecond laser pulses. Las. Phys., 2006; 16: 336-339.

\footnotetext{
* Contribuição técnica ao $69^{\circ}$ Congresso Anual da ABM - Internacional e ao 14ํㅡㄹ ENEMET - Encontro Nacional de Estudantes de Engenharia Metalúrgica, de Materiais e de Minas,21 a 25 de julho de 2014, São Paulo, SP, Brasil.
} 
28 Samad RE., Baldochi SL and Vieira Junior ND. Diagonal scan measurement of Cr:LiSAF 20 ps ablation threshold,"Appl. Opt. 2008; 47: 920-924.

29 Liu JM. Simple technique for measurements of pulsed Gaussian-beam spot sizes. Opt. Lett. 1982; 7: 196-198.

30 Machado LM., Samad RE., Rossi W, and Vieira Junior ND. D-Scan measurement of ablation threshold incubation effects for ultrashort laser pulses. Opt. Expr. 2012; (20): 4114-4123.

31 Morada M, Kamal S, Englezos P, Hatzikiriakos GS. Femtosecond laser irradiation of metallic surfaces: effcts of laser parameters on superhydrophobicity. Nanotecnology. 2013; 24

32 Barada KN, Mool CG. Ultrafast laser-induced self-organized conical micro/nano surface structures and their origin. Optics and Lasers in Engineering. 2010; 48: 966-973.

* Contribuição técnica ao $69^{\circ}$ Congresso Anual da ABM - Internacional e ao 14ํㅡㄹ ENEMET - Encontro Nacional de Estudantes de Engenharia Metalúrgica, de Materiais e de Minas,21 a 25 de julho de 2014, São Paulo, SP, Brasil. 